\title{
Les trois institutions oratoires à l'usage de Henri III : un compendium des traditions latine, hellénistique et humaniste ${ }^{1}$
}

\author{
CLAUDE LA CHARITÉ \\ Université du Québec à Rimouski
}

The literary tradition has preserved three Artes Rhetoricae written for the last of the Valois kings, who reigned in France under the name of Henry III from 1574 to 1589. These three texts are Jacques Davy Du Perron's Avant-discours de rhetorique, ou Traitté de l'eloquence, Jacques Amyot's Projet de l'eloquence royale, and Germain Forget's Rhetorique françoise faicte particulierement pour le roy Henry 3. All three very likely originated as academic speeches pronounced at the Louvre, in the presence of Henry III, in the final sessions of the Palace Academy during the summer of 1579. This article offers a re-reading of the three treatises in order to situate them in the history of rhetoric. It aims to show how each author collects and presents teachings of the principal rhetorical traditions. Thus, Du Perron, inspired mainly by Quintilian and Cicero, proposes a kind of abridged version of the rhetorical thought of Latin Antiquity. Amyot, for his part, puts forth a synthesis of ancient Greek rhetorical theory starting with Plutarch, Dyonisius of Halicarnassus and Demetrius of Phalerus. Germain Forget provides an account of Renaissance innovations, by adopting the nomenclature of Peter Ramus under the rubric of elocutio. The objective of this essay is to shed light on the complementary nature of the three treatises, as well as to suggest a probable order in which they were presented to the King, following a logical gradation from the most general to the most specific.

$\mathrm{L}$ a tradition nous a conservé trois rhétoriques écrites à l'usage du dernier des Valois qui a régné sur la France, sous le nom de Henri III, de 1574 à 1589 : l'Avantdiscours de rhetorique, ou Traitté de l'eloquence de Jacques Davy Du Perron, le Projet de l'eloquence royale de Jacques Amyot et la Rhetorique françoise faicte particulierement pour le roy Henry 3 de Germain Forget. Malgré la réédition intégrale du traité d'Amyot en $1992^{2}$ et la reprise de quelques extraits du traité de Du Perron dans l'anthologie L'art de parler en $2003^{3}$, personne n'a, à ce jour, consacré d'étude à cet ensemble de textes qui révèlent pourtant la persistance de l'idéal humaniste de la toute-puissance de la parole éloquente, même au plus fort des guerres civiles. 
Robert J. Sealy, qui a proposé une brève paraphrase des trois rhétoriques dans son ouvrage phare, The Palace Academy of Henry III 4 (1981), présume que les trois textes auraient été en fait des discours académiques prononcés au Louvre, en présence de Henri III, lors des dernières séances de l'Académie du Palais, sans doute au début de l'été 1579 et, à coup sûr, avant la surdité partielle du roi survenue au début de septembre de la même année. Ces trois discours sur le thème de l'éloquence constituent un cas à part dans l'enseignement de l'Académie du Palais fondée en janvier 1576, dans la mesure où toutes les séances précédentes avaient été consacrées à la philosophie : philosophie naturelle, logique et philosophie morales . Si le rattachement de ces trois textes à l'Académie du Palais est problématique, il n'en demeure pas moins qu' ils s'inscrivent à coup sûr dans une préoccupation constante du roi depuis son départ de Pologne et son accession au trône de France, à savoir exercer lui-même le magistère de la parole politique dans son royaume. Son bref règne sur le trône des Jagellon en Pologne, de janvier à juin 1574, l'avait contraint à s'en remettre presque entièrement aux humanistes de son entourage, dans la mesure où la langue officielle de cette république aristocratique était le latin, langue que le monarque comprenait à peine ${ }^{6}$. Cette expérience frustrante l'avait cependant convaincu de la nécessité pour un prince d'exercer lui-même son autorité par la parole pour pouvoir mieux arbitrer les différends entre les grands, rôle qu'il continuera à jouer même après son retour en France et malgré la profonde différence de nature des institutions politiques des deux royaumes. C'est bien à ce désir de ne plus s'en remettre seulement à ses mandataires que cherchent à répondre les trois rhétoriciens, en particulier Jacques Amyot qui, dans son chapitre IX « De quoi parle un Prince en temps d'affaires $\gg$, écrit :

[... ] la plupart des princes ne cherchent point ces entrevues et parlements, mais traitent des affaires parl'entremise de leurs ambassadeurs, qui sont communément gens choisis, sçavans et éloquens. Par quoi le Prince qui leur sait bien répondre en rapporte grande louange[.] (PER, p. 75)

À ce titre, les trois rhétoriques de 1579 peuvent être vues comme la clef de voûte de toute cette formation humaniste que Henri III chercha à acquérir à partir de l'obtention de sa deuxième couronne. Nous voudrions proposer une lecture de ces trois traités qui, selon l'expression de Salazar dans son édition de 1992, méritent « une analyse conjuguée ${ }^{7} \gg$, dans la perspective de l'histoire de la rhétorique, pour montrer comment chacun des trois auteurs, à sa manière, recueille les leçons des principales traditions rhétoriques, pour offrir au roi une sorte d'épitomé de la réflexion de l'Antiquité latine, de l'Antiquité grecque et de la Renaissance ${ }^{8}$. Pour ce faire, nous suivrons une progression du plus général au plus particulier qui correspond sans 
doute à l'ordre dans lequel les discours furent prononcés à l'Académie : d'abord, le traité de Du Perron, ensuite, celui d'Amyot et, enfin, celui de Germain Forget ${ }^{9}$.

\section{L'Avant-discours de rhetorique, ou Traitté de l'eloquence de Jacques Davy Du Perron ou le Dialogue des orateurs, le De Oratore et l'Institution oratoire revisités}

De fait, l'Avant-discours de rhetorique, ou Traitté de l'eloquence de Jacques Davy Du Perron (1556-1618) constitue le plus général des trois discours académiques conservés. Introduit à la Cour en 1576, converti au catholicisme en 1577, Du Perron, au moment où il présente son traité à Henri III, n'a pas encore entamé sa carrière de prélat. C'est en 1591 qu'il sera nommé évêque d'Évreux et en 1604 qu'il sera élevé à la dignité de cardinal ${ }^{10}$. Il passait pour un convertisseur extrêmement efficace grâce à son éloquence hors pair. Dans son journal, Pierre de l'Estoile relate la célèbre anecdote sur l'aptitude de Du Perron à prouver aussi bien l'existence de Dieu que son inexistence :

Le vendredi $25^{\mathrm{e}}$ de ce mois [de novembre 1583 ], advinst, au disner du Roy, que mons ${ }^{\mathrm{r}}$ Du Perron, grand discoureur et philosophe, et que le Roi oioit volontiers comme faisant cas de son esprit et de sa memoire, fit un brave discours contre les atheistes et comme il y avoit un Dieu, et le prouva par des raisons si claires, evidentes et à propos qu'il sembloit bien n'y avoir lieu aucun d'y contredire : à quoi le Roy monstra qu'il avoit pris plaisir et l'en loua. Mais Du Perron, s'oubliant comme font ceux de son humeur, va dire au Roy : «Sire, j’ai prouvé aujourd'hui, par bonnes raisons tresbonnes et evidentes, qu'il y avoit un Dieu : demain, Sire, s'il plaist à Vostre Majesté donner encores audience, je prouverai par raisons aussi bonnes et evidentes qu'il n'y a point du tout de Dieu ${ }^{11}$. »

C'est à coup sûr cette maestria oratoire qui désignera $\mathrm{Du}$ Perron comme rédacteur de la harangue prononcée par Henri III aux États Généraux de $1588^{12}$.

Comme le suggère le titre d'avant-discours, que l'on trouve dans les OEuvres diverses de 1633, il s'agit d'une sorte d'introduction aux deux autres discours, en ce sens qu'il se contente de poser le cadre théorique général qui rendra possible ensuite l'enseignement proprement dit de la rhétorique. Le texte aurait été publié en 1607 sous le titre Discours de l'excellence de l'eloquence par le sieur L. D. G., prononcé en l'Academie du sieur P. Bien qu'aucun exemplaire de cette édition ne soit aujourd'hui connu, c'est sur la base du sous-titre « prononcé en l'Academie du sieur P. [=Pibrac] » que Sealy date le traité de 1579 et l'associe aux travaux de l'Académie du Palais ${ }^{13}$. 
Du Perron divise son Avant-Discours en quatre grandes sections : 1) l'utilité et la dignité de la rhétorique ; 2) la facilité ou la difficulté de la rhétorique ; 3) s'il y a une science et un art de l'éloquence ou bien si elle dépend de la nature ; 4) le genre et la définition de la rhétorique.

En fait, le plan du traité de Du Perron recoupe les chapitres xv à xix du livre II de l'Institution oratoire (ca 96 ap. J.-C.) de Quintilien. La section 1, l'utilité et la dignité de la rhétorique, décalque le chapitre xvi « Si la rhétorique est utile ». La section 3 , s'il y a une science et un art de l'éloquence ou bien si elle dépend de la nature, s'inspire des chapitres xvii « Si la rhétorique est un art $\gg$ et xix « Lequel, de l'art ou de la nature, joue le rôle le plus important dans l'éloquence $\gg$. La section 4 , le genre et la définition de la rhétorique, fait écho au chapitre xviii « Dans quel groupe d'arts rentre la rhétorique » pour ce qui est du genre et au chapitre xv « Qu'est-ce que la rhétorique et quelle est sa fin $\gg$ pour ce qui touche la définition.

Commençons par la première section consacrée à l'utilité et à la dignité de la rhétorique. C'est sans doute sur ce plan que la pensée de Du Perron apparaît la plus intéressante, dans la mesure où ce dernier y propose une analyse sociopolitique qui, d'une certaine manière, prolonge et nuance le fameux Dialogue des orateurs (107 ap. J.-C.) de Tacite, un peu à la manière de ce que fera, un siècle plus tard, le père René Rapin dans les Réflexions sur l'usage de l'éloquence de ce temps (1672).

En fait, Tacite semble conclure à la complète déchéance de la rhétorique en son temps qui « conserve à peine l'usage du nom même d'Orateur ${ }^{14}$ ». Dans le Dialogue des orateurs (XXXVI), c'est le personnage de Secundus qui met en cause les conditions politiques pour expliquer ce déclin de l'art oratoire, comme si la rhétorique ne pouvait plus s'épanouir dans un régime autoritaire tel que le principat :

Semblable au feu, la grande éloquence tire sa force de ses alimens; elle s'accroît par le mouvement et par l'agitation, et d'un foyer brûlant elle jette une vive lumière. C'est ainsi que l'éloquence des anciens fit tant de progrès ; car, bien que les Orateurs de notre temps aient aussi obtenu ce qu'il étoit convenable de leur accorder sous un gouvernement heureux et tranquille, cependant on voyoit leurs prédécesseurs tirer plus de fruit des troubles et de la licence, lorsque, dans la confusion des pouvoirs, l'Etat manquoit d'un chef unique, et que chaque Orateur étoit aussi habile qu'il pouvoit le faire croire à une multitude facile à égarer : de-là cette promulgation continuelle de nouvelles loix, sous le nom du peuple ; de-là ces harangues fréquentes de Magistrats qui passoient des nuits presque entières dans la tribune de la place publique ; de-là ces accusations intentées contre des hommes puissans, et des inimitiés vouées encore à leurs maisons ; de-là des factions formées par des grands, et de perpétuelles contestations entres les Sénateurs et les Plébéiens, qui touts, à la vérité, déchiroient la république, mais qui exerçoient l'éloquence de ces temps-là et la combloient de récompenses d'un grand $\operatorname{prix}^{15}[$.] 
Du Perron reprend la question dans le contexte du règne de Henri III. D’entrée de jeu, il concède volontiers que la rhétorique, en particulier délibérative, est plus appropriée à un régime républicain :

[ ... ] les lieux où l'Eloquence a tousjours regné et triomphé, ça esté aux Republiques et gouvernements populaires, à raison qu'en ces Estats la, ausquels les deliberations dépendent de la multitude, le premier poinct qu'il faut recercher, c'est que le peuple, qui est, comme on dit, un animal à plusieurs testes, convienne en une mesme intention ${ }^{16}[$.

Il reconnaît toutefois que la rhétorique délibérative a également sa place dans une monarchie, même s'il envisage d'abord le rôle du monarque plutôt en tant que destinataire qu'en tant qu'orateur délibératif, ce qui est assez dire le niveau de généralité de cet avant-discours (les deux autres traités ne s'intéressant qu'à la rhétorique royale et au rôle du monarque comme orateur) :

[... ] aussi aux Royaumes et Monarchies, à cause que les deliberations y dépendent de la volonté d'un seul, qui est ordinairement plus instruict et preparé de l'estat des choses que ne sçauroit estre une multitude grossiere et ignorante; et par ainsi ne se laisse pas charmer si aisément aux paroles, mais veut voir et découvrir le fond des affaires, elle n'y a pas tant de pouvoir. (ADR, p. 760)

Puis, Du Perron en vient à envisager le rôle actif du monarque ou de ses officiers dans l'exercice de la parole éloquente, office de tout roi, quelles que soient les circonstances :

Il y a les ambassades vers les Princes estrangers, il y a les propositions au Conseil d'Estat, il y a les relations, quand on vient rendre compte de quelque longue commission qu'on exerce; il y a les harangues qu'on peut prononcer aux Parlements, aux principaux sieges des Provinces, lors qu'on y prend possession de quelque gouvernement ou de quelque authorité[.] (ADR, p. 760)

Enfin, l'académicien s'attarde sur le contexte spécifique du règne de Henri III, à savoir le rôle de la rhétorique en pleines guerres de Religion :

[ ... ] ily a celles [=les harangues] qu'on peut faire au corps de ville, et aux assemblées du peuple, et principalement en une saison telle qu'est la nostre, c'est à dire, durant les guerres civiles, menées par des pretextes populaires, comme pour la Religion. Car alors le peuple veut prendre cognoissance de cause, et estre luy mesme rendu capable de l'estat des affaires, et sur ces occasions ceux qui montent aux chaires publiques, et se sçavent aider des armes de l'eloquence, peuvent beaucoup pour le precipiter aux desordres, insolences et confusions, ou pour l'en rappeler et divertir. (ADR, p. 760) 
Du Perron définit ici un champ d'action plus vaste que celui de l'éloquence royale en temps de paix. Même s'il ne dit pas explicitement que le roi se doit de descendre dans l'arène délibérative pour riposter à ses adversaires, sur le même terrain avec les mêmes armes pour rappeler et divertir le peuple des désordres, insolences et confusions, il ne semble pas du tout l'exclure, en évoquant les « assemblées du peuple » qui englobent sans doute les États Généraux, même si ce rôle d'orateur délibératif de la part d'un roi très chrétien a pu scandaliser certains contemporains, comme Marc Fumaroli le rappelle dans son Âge de l'éloquence (1980) : « Le pire scandale avait été peut-être, pour Montaigne comme pour Pasquier, de voir Henri III donner l'exemple, et entrer dans la lice délibérative ${ }^{17}$. $\gg$ On voit bien ici que ce rôle politique exceptionnel n'était pas qu'une lubie du dernier des Valois, mais qu'il était encouragé par certains conseillers avisés comme Du Perron. En fait, il recommande à Henri III de s'inscrire dans le prolongement de la contre-offensive catholique inaugurée sous le règne de Charles IX, avec, entre autres, les Discours des Miseres de ce temps (1562-1563) de Ronsard ${ }^{18}$.

Du Perron ne défend pas l'utilité de la rhétorique seulement au nom du « maniement des grandes affaires $\gg$, mais aussi au nom de la parole comme propre de l'homme :

[ ... ] quand toutes ces considerations ne nous toucheroient aucunement $[\ldots]$ et que nous ne mesurerions point l'utilité de l'eloquence à l'avantage qu'elle peut apporter pour le maniement des grandes affaires, encore en recueillons-nous un assez doux fruict, du contentement que nous recevons en nous mesmes, en parlant et discourant ensemble, et rendant nostre compagnie aggreable à ceux avec qui nous avons à converser, ou bien en escrivant les conceptions de notre esprit, soit pour les communiquer à nos amis, soit pour les consacrer à l'immortalité ; attendu que la seule possession d'une si belle science, sans laquelle toutes choses seroient muettes et privées de la lumiere des siecles presents et de la memoire de la posterité, se sert de digne et suffisante recompense à elle-mesme. (ADR, p. 760-761)

S'en suit un développement qui résume l'argumentaire du chapitre xvi du livre II de l'Institution oratoire de Quintilien, au point de traduire littéralement le passage sur l'ingéniosité des animaux qui apparaissent cependant dénués de raison, faute de parole : 
Avant-discours de rhetorique, ou Traitté de l'eloquence (1579) de Jacques Davy Du Perron [... ] l'industrie qu'ont les animaux de bastir leurs nids avec tant d'artifice, d'elever leurs petits, d'amasser des provisions pour l'hyver, et mesmes de faire des ouvrages qui sont du tout inimitables, comme le miel et la cire; il semble que ce soient choses qui participent de la raison; et qu'il y a quelque rayon de lumiere d'intelligence en ceux qui les font: Mais d'autant que l'usage de la parole leur est interdict, nous les appellons muets et irraisonnnables. (ADR, p. 761)
Institution oratoire (ca 96 ap. J.-C.) de Quintilien (II, xvi, 16)

Nam [ ... ] nidos texere et educare fetus et excludere, quin etiam reponere in hiemem alimenta, opera quaedam nobis inimitabilia, qualia sunt cerarum ac mellis, efficere nonnullius fortasse rationis est, sed quia carent sermone quae id faciunt, muta atque inrationalia vocantur ${ }^{19}$.

Dans la seconde section du traité, réservée à la facilité ou à la difficulté de la rhétorique, Du Perron délaisse Tacite et Quintilien pour suivre étroitement le livre I du De Oratore (55 av.J.-C.) de Cicéron. On pourrait multiplier les exemples de traduction littérale, mais nous nous contenterons d'un seul passage, celui où Cicéron met en rapport la rareté des excellents orateurs et le grand nombre d'éminents politiques :

Avant-discours de rhetorique, ou Traitté de l'eloquence (1579) de Jacques Davy Du Perron

[... ] d'hommes d'Estat, et qui sçachent bien juger et ordonner des affaires, combien en avons nous veu de nostre temps, et combien plus encore de la memoire de nos peres, et de celle de nos predecesseurs? là où d'excellents Orateurs, il s'est coulé souventesfois beaucoup de siecles, sans qu'on en ayt remarqué un seul : et de passables et mediocres, ç'a été quand chaque âge a produit le sien. (ADR, p. 762)
De Oratore ( 55 av. J.-C.) de Cicéron $(\mathrm{I}, \mathbf{8})$

Iam vero, consilio, ac sapientia qui regere ac gubernare rem publicam possint, multi nostra, plures patrum memoria atque etiam maiorum exstiterunt, cum boni perdiu nulli, vix autem singulis aetatibus singuli tolerabiles oratores invenirentur ${ }^{20}$.

Dans cette section, seule la conclusion s'éloigne de la source cicéronienne. Alors que, dans le De Oratore (I, 22), le champ d'exercice de l'éloquence est restreint « aux plaidoyers du Barreau, et aux deliberations du Senat ${ }^{21}$ », Du Perron quant à lui élargit le domaine rhétorique, en y annexant l'éloquence de la chaire, la conversation, l'écrit en général et la correspondance en particulier :

Il y a, comme nous avons dit, les harangues et oraisons publiques, dont les Gentilshommes un peu advancez en la cognoissance des affaires, se peuvent mesler ; il y a les plaidoyez et les sermons, que je laisse aux Advocats et aux gents d'Eglise ; il y a les propos communs, soit avec ceux de nostre sexe, soit avec les honnestes femmes ; il y a les discours par escrit, il y a les lettres familieres à nos amis. (ADR, p. 764)

Cette extension du domaine de la rhétorique suppose d'étudier la pratique oratoire du roi, non seulement dans ses manifestations les plus officielles comme 
les harangues prononcées aux États Généraux, mais également dans ses pratiques plus « privées » comme les lettres familières, ce qui multiplie les possibilités d'analyse compte tenu de la colossale correspondance du monarque toujours en cours d'édition ${ }^{22}$.

Dans la troisième section relative à l'éloquence comme art ou nature, Du Perron définit les quatre concepts fondamentaux qui font de la rhétorique un domaine qui «se peut dompter par regles et par preceptes $\gg(A D R$, p. 765$)$ et donc un savoir susceptible d'être enseigné et appris. Ces quatre pierres angulaires de la pédagogie de l'éloquence sont la natura, l'ars, l'imitatio et l'exercitatio : « Il [=l'Orateur] constituëra donc la perfection de l'eloquence en ces quatre poincts, comme aux quatre moyens et degrez par lesquels elle est acquise, asçavoir en la bonté de la nature, en la cognoissance de l'art et des preceptes, en l'imitation des excellents ouvriers, et en l'usage ou exercice $\gg(A D R$, p. 769).

Dans cette section, Du Perron résume une fois de plus l'Institution oratoire, en traduisant littéralement certains passages, notamment l'exemplum des bœufs ardents d'Annibal pour montrer que le faux présenté comme vrai n'est pas l'apanage des orateurs, mais se trouve aussi chez les tacticiens militaires :

\footnotetext{
Avant-discours de rhetorique, ou Traitté de

Institution oratoire (ca 96 ap. J.-C.)

l'eloquence (1579) de Jacques Davy Du Perron de Quintilien (II, xvii, 19)

[... Annibal, quand il fit attacher la nuict des fagots allumez à la teste des bœufs de son armée, et leur donna la chasse à travers les montagnes, afin de faire penser à ses ennemis, que c'est son camp qui delogeoit, et cependant trouver moyen de sortir d'un mauvais passage, n'ignoroit pas la fausseté de ceste apparence, ny la ruse de son stratageme. (ADR, p. 761)

De la même manière, Du Perron reprend souvent mot à mot les analogies que Quintilien multiplie entre la rhétorique et d'autres arts tels que la médecine ou la navigation :

\begin{tabular}{ll}
$\begin{array}{c}\text { Avant-discours de rhetorique, ou Traitté de } \\
\text { l'eloquence (1579) de Jacques Davy Du Perron }\end{array}$ & \multicolumn{1}{c}{$\begin{array}{c}\text { Institution oratoire (ca 96 ap. J.-C.) } \\
\text { de Quintilien (II, xvii, 24-25) }\end{array}$} \\
\hline$[\ldots]$ tout ainsi que le Pilote, nonobstant qu'il se & Nam et gubernator vult salva nave in portum \\
propose bien de conduire son vaisseau à bon port, & pervenire ; si tamen tempestate fuerit abreptus, \\
toute-fois s'il est emporté et vaincu par la tem- & non ideo minus erit gubernator $[\ldots]$ Et medicus \\
peste, il n'en est pas moins Pilote, ny le Medecin & sanitatem aegri petit ; si tamen aut valetudinis vi \\
semblablement, encore que l'imbecillité de la & aut intemperantia aegri aliove quo casu summa \\
nature, ou l'excés et l'intemperance de son malade, & non contingit [ ... ] medicinae fine non excidet ${ }^{24}$. \\
l'empesche de faire son effet. $(A D R, p .766)$ &
\end{tabular}

[ ... ] Hannibal, cum, inclusus a Fabio, sarmentis circum cornua boum deligatis incensisque per noctem in adversos montes agens armenta, speciem hosti abeuntis exercitus dedit, sed illum fefellit, ipse quid verum esset non ignoravit ${ }^{23}$.
} 
Même le développement de Du Perron sur les rapports complémentaires de la natura et de l'ars, alors que pourtant il fait intervenir un artiste de la Renaissance, en l'occurrence Michel Ange, n'est qu'une adaptation du chapitre xix de l'Institution oratoire où Quintilien évoquait, lui, l’art de Praxitèle :

Avant-discours de rhetorique, ou Traitté de l'eloquence (1579) de Jacques Davy Du Perron

$[\ldots]$ si Michell'Ange, ou quelqu'autre excellent sculpteur, avoit mis en œuvre une de ces pierres dont on fait les meules; il n'y a celuy qui n'aimast beaucoup mieux une piece de marbre toute simple : Mais s'il avoit employé le mesme labeur sur du marbre, ou de l'albastre, alors on y priseroit trop plus l'ouvrage, que la matiere. (ADR, p. $767-768$ )
Institution oratoire (ca 96 ap. J.-C.) de Quintilien (II, xix, 3)
Et si Praxiteles signum aliquod ex molari lapide conatus esset exculpere, Parium marmor mallem rude ; at si illud idem artifex expolisset, plus in manibus fuisset quam in marmore ${ }^{25} .[\ldots]$

Dans la dernière section dévolue au genre et à la définition de la rhétorique, Du Perron inscrit sa réflexion une fois de plus dans le sillage de Quintilien. La typologie qu'il dresse des types d'art est traduite du chapitre xviii de l'Institution oratoire :

\section{Avant-discours de rhetorique, ou Traitté de l'eloquence (1579) de Jacques Davy Du Perron}

[... ] des arts ou des sciences (car icy nous les prenons confusément en un mesme sens,) les unes consistent en une simple cognoissance d'intelligence des choses, comme est l'astronomie ne se proposants aucune action, mais se contentants de la seule contemplation de leur sujet; et celles-la s'appellent contemplatives : les autres s'estendent bien à l'action, mais ne recherchent rien plus outre, et ne laissent aucun effet apres l'operation, comme est la danse et le jeu des instruments de musique ; et celles-la sont dittes sciences actives : les troisiémes tendent à produire quelque effect qui puisse estre exposé à la veuë, et demeurer apres l'action, comme la sculpture et la peinture ; et ces dernieres se nomment effectives. Ceste division donc ainsi persupposée, nous pouvons dire que la Rhetorique appartient proprement à l'ordre des sciences actives, combien qu'elle participe en quelque sorte de toutes les trois.(ADR, p. 769)
Institution oratoire (ca 96 ap. J.-C.) de Quintilien (II, xviii, 1-3)

Cum sint autem artium aliae positae in inspectione, id est cognitione et aestimatione rerum, qualis est astrologia, nullum exigens actum, sed ipso rei cujus studium habet intellectu contenta,

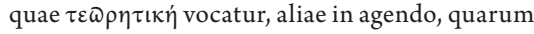
in hoc finis est et ipso actu perficitur nihilque post actum operis relinquit, quae $\pi \rho \alpha \kappa \tau \iota \kappa \eta \dot{~ d i c i t u r, ~}$ qualis saltatio est, aliae in effectu, quae operis, quod oculis subjicitur, consummatione finem accipiunt, quam $\pi$ oı $\tau \iota \kappa \eta ́ v$ appellamus, qualis est pictura. Fere judicandum est rhetoricen in actu consistere ; hoc enim, quod est officii sui, perficit, atque ita ab omnibus dictum est. Mihi autem videtur etiam ex illis ceteris artibus multum adsumere $^{26}[$.

Enfin, pour ce qui est de la définition de la rhétorique, Du Perron résume de manière extrêmement concise le chapitre XV de Quintilien, en opposant une définition qui met en valeur la dimension persuasive et une définition fondée sur la dimension éthique de la rhétorique : 
Pour le regard de la definition qu'on luy peut donner, il y en a plusieurs qui luy ont esté attribuées par les Anciens, lesquelles vouloir toutes toucher icy l'une apres l'autre, ce seroit une curiosité plus ambitieuse que necessaire : et partant il nous suffira d'en choisir icy deux des plus specieuses : l'une que la Rhetorique est la science de bien parler, l'autre que c'est la science de bien dire ; dont la premiere est prise du but et de la fin de l'eloquence, la seconde est tirée de sa charge et de son office. (ADR, p. 769-770)

La concision est ici telle qu'elle nuit à la clarté du propos. Il est probable toutefois que Du Perron cherche ici à opposer une définition fondée sur la persuasion, la science de bien parler ou ars dicendi ad persuadendum, et une définition fondée sur l'exigence éthique que l'orateur soit un homme bon, la science de bien dire ou ars bene dicendi, qui est celle de Quintilien. La conclusion de Du Perron vise à faire la synthèse des deux : «la Rhetorique est un art de bien et proprement parler de tout sujet proposé, et a pour sa matiere toutes les choses vray-semblables, qui peuvent tomber en discours entre les hommes $\gg$ (ADR, p. 770).

Pour parvenir à cette conclusion, le rhétoricien emprunte certains exemples à Quintilien pour prouver qu'il n'y a pas que les paroles qui persuadent afin de rejeter la définition fondée exclusivement sur la persuasion :

Avant-discours de rhetorique, ou Traitté de l'eloquence (1579) de Jacques Davy Du Perron

$[\ldots]$ un ancien Orateur défendant un citoyen Romain, qui estoit luy mesme present, et assistoit au jugement de la cause, luy déchira ses vestements en plein Senat, et découvrit les playes qu'il avoit autresfois receuës en l'estomach pour la protection de la Republique ; faisant par ce moyen plus de force et de violence aux yeux qu'aux oreilles du peuple Romain ; lequel avec ses artifices, il émeut à ressentiment et à compassion. [ ... ] une courtisane Grecque, nommée Phryne, estant poursuivie en jugement, ne s'exempta pas tant du supplice par la harangue d'un sien Advocat, nommé Hyperides, encores qu'elle fust admirable, comme par l'invention qu'elle trouva d'ouvrir un des costez de sa robbe, et faire paroistre à nud une partie de son corps qui est tout doüé d'une extréme beauté. ( $A D R$, p. 770)
Institution oratoire (ca 96 ap. J.-C.) de Quintilien (II, xv, 7-9)

Nam et Manium Aquilium defendens Antonius, cum scissa veste cicatrices, quas is pro patria pectore adverso suscepisset, ostendit, non orationis habuit fiduciam, sed oculis populi Romani vim attulit ; quem illo ipso aspectu maxime motum in hoc, ut absolveret reum, creditum est. [ ... ] Et Phrynen non Hyperidis actione, quamquam admirabili, sed conspectu corporis, quod illa speciosissimum alioqui diducta nudaverat tunica, putant periculo liberatam ${ }^{27}$.

L'Avant-Discours de Du Perron, tout en résumant et en adaptant la tradition latine de la rhétorique, en particulier le Dialogue des orateurs de Tacite, le De Oratore de Cicéron et surtout le livre II de l'Institution oratoire de Quintilien, s'en tient à des considérations purement théoriques, sans jamais évoquer de préceptes qui 
fonderaient l'art rhétorique et encore moins d'exemples bien concrets d'éloquence qui conviendrait à un roi comme Henri III. Il balise plutôt le terrain, en montrant que la rhétorique est non seulement utile dans une monarchie, mais qu'elle est même indispensable en période de guerres civiles, que la rhétorique, même si elle suppose une nature éloquente, est un art susceptible de perfectionner cette éloquence naturelle grâce à la pratique et à l'imitation.

\section{Le Projet de l'eloquence royale de Jacques Amyot ou les « preceptes generaux » tirés de la tradition hellénistique}

Le Projet de l'eloquence royale de Jacques Amyot est le mieux connu des trois traités. Il est resté manuscrit jusqu'à l'édition qu'en a procurée Philippe-Denis Pierres (1741-1808) en 1805 d'après un manuscrit autographe aujourd'hui perdu ${ }^{28}$. C'est ce texte que Salazar a reproduit, en le modernisant légèrement, dans la collection «Le corps éloquent » aux Belles Lettres en 1992. Jacques Amyot (1513-1593) n’a pas besoin d'être présenté, tant il est connu comme traducteur de Plutarque dont il traduisit aussi bien les Vies parallèles (1559) que les OEuvres morales (1572). Après avoir été lecteur de grec à l'Université de Bourges grâce à l'intervention de Jean de Morvilliers, lieutenant général de Berry et auteur présumé de la harangue prononcée par Henri III aux États Généraux de $1576^{29}$, Amyot fut nommé en 1557 précepteur et aumônier des enfants de France (les futurs Charles IX et Henri III), puis, en 1560, grand aumônier de France et, enfin, évêque d'Auxerre en $1570^{30}$.

Dans le prolongement de l'Avant-Discours, Amyot s'attache à donner ce que Du Perron appelle les « preceptes [ ... ] generaux $\gg(A D R$, p. 768) de l'art rhétorique, ce que du reste Amyot dit clairement au chapitre VII : « vous trouverez ici les premières règles et préceptes principaux de l'art » (PER, p. 73).

L'ensemble du traité est parcouru par l'idéal de la paideia grecque et insiste sur la culture générale que présuppose toute éloquence : « ceux qui ont enseigné l'art de bien dire ne l'avoir autrement formé qu'avec la connoissance des belles sciences, sans lesquelles ce qu'on appelleroit éloquence ne seroit à la vérité qu'une baverie indiscrète et ignorante $\gg(P E R$, p. 71-72). Ce qui, bien sûr, n'est pas sans rappeler d'une part la définition même de la rhétorique par Quintilien, ars bene dicendi, mais aussi l'ambition au fondement de l'Institution oratoire, l'éloquence, au chapitre X du livre I, supposant l'assimilation de « ce cercle de sciences que les Grecs nomment Encyclopedie ${ }^{31} \gg$.

C'est ainsi qu'Amyot est amené à traiter succinctement de l'invention et des lieux oratoires au chapitre $\mathrm{X} \ll$ Des lieux d'où l'on titre les arguments et les 
passions $\gg$, de la disposition et des parties du discours au chapitre XI, de l'élocution et de l'agencement des mots aux chapitres XII et XIII, de la mémoire et de l'action au chapitre XIV.

Cela étant, c'est surtout dans les premiers chapitres que l'on a vu l'originalité de la pensée d'Amyot, où il s'attache à définir une éloquence proprement royale. Cette originalité est cependant toute relative dans la mesure où, en réalité, l'évêque d'Auxerre, dans cette première partie de son traité, se contente surtout de recueillir les leçons de Plutarque, en particulier de l' « Instruction pour ceulx qui manient affaires d'estat $\gg$ qu'il avait traduite en 1572 .

Dans son premier chapitre, Amyot cherche à montrer la supériorité de l'éloquence sur la force dans l'art de gouverner les hommes et évoque à cette fin l'exemplum de Thucydide, vainqueur de Périclès à la lutte, mais vaincu à son tour par l'éloquence de ce dernier. Or, cet exemplum est repris presque mot à mot de la traduction du traité moral de Plutarque, comme on peut en juger en confrontant les deux textes :

\section{Jacques Amyot, \\ Projet de l'eloquence royale (1579)}

\section{Plutarque, « Instruction pour ceulx qui manient affaires d'estat », trad. Jacques Amyot (1572)}

Thucydide $[\ldots$ f.. fut quelquefois enquis par le Roi Thucydides [ ... ] qui estant un jour enquis par Archidamus, qui étoit plus adroit à la lutte, lui ou Périclès : cela seroit, dit-il, difficile à juger, car aussitôt que je l'ai porté par terre avec l'adresse et force de ma personne, il fait accroire par son bien dire à ceux qui l'ont vu qu'il n'est pas tombé, et le gagne sur moi par le plat de la langue. (PER, p. 44)

le roy de Lacedemone Archidamus lequel estoit le plus adroit à la luicte de luy ou Pericles : Cela respondit il seroit bien mal-aisé à dire : car quand je l'ay porté par terre en luictant, luy en disant persuade aux assistans qui l'ont veu, qu'il n'est pas tombé, et le gaigne ${ }^{32}[$.

Au chapitre III, Amyot oppose deux types d'éloquence, une éloquence vulgaire, comparée à une courtisane, et une éloquence noble, vraie dame d'honneur, qui est le propre de l'éloquence royale :

Il y a deux manières d'éloquence aisées à remarquer chez les anciens orateurs ; l'une pleine de babil et d'afféterie ainsi qu'une courtisane : l'autre ornée d'un parler doucement grave, d'un port, grâce et beauté naïve, comme une femme d'honneur. (PER, p. 50)

Cette opposition entre une éloquence vulgaire et une éloquence noble est courante dans les traités hellénistiques. Denys d'Halicarnasse, dans Les orateurs antiques ( $\mathrm{I}^{\mathrm{er}}$ siècle av. J.-C.), compare l'ancienne éloquence, amie de la sagesse, à une épouse bien née, alors que la nouvelle éloquence méprisable, déclamatoire, déréglée, étrangère à la philosophie et à tous les arts libéraux, est assimilée à une courtisane, dans la maison de débauche qu'est devenue la Grèce $(I, i, 5): \ll$ Dans ces demeures, l'épouse de naissance libre, à la conduite sage, n'est maîtresse d'aucun de ses biens 
tandis que la courtisane aux mœurs légères, qui n'est là que pour ruiner la maison, exige de régner sur toute la fortune, ne manifestant que mépris pour l'épouse et la terrorisant $^{33} \gg$.

Chez Plutarque, bien que la métaphore soit différente, l'enseignement est identique, qui oppose une éloquence vulgaire de charlatan à une grave éloquence digne d'un père de famille :

Puis qu'il est donc ainsi, que le principal instrument d'un sage gouverneur est la parole, il fault tout premierement qu'elle ne soit point affettee, ny pompeuse et fardee, comme seroit celle de un jeune charlatan [ ... ] [il faut que] soit son parler plein d'une affection naïfve, d'une vray[e] magnanimité, d'une franchise de remonstrance paternelle, qu'il sente son pere du public, plein de bon sens, de provoyance soigneuse, aiant la grace attraiante conjoincte avecl'honeste dignité, en termes graves, raisons pertinentes, et vray semblables[.] (oM, fo $\left.234, v^{\circ}\right)$

Au chapitre IV, Amyot avance que l'éloquence royale consiste en deux principales parties : «l'entendement et la parole ». Or, Plutarque ne dit pas autre chose dans son traité, qui, par ailleurs, insiste sur la supériorité d'un gouvernant qui, plutôt que de déléguer la parole, l'assume lui-même, ce qui répondait précisément au désir de Henri III après la déception de son règne polonais :

[ ... ] le bon gouverneur d'estat doit avoir dedans soy-mesme l'entendement qui manie le timon, et puis la parole qui fait entendre sa volonté, à fin qu'il n'ait point affaire à tout propos de la voix d'un autre[.] (oM, $\left.\mathrm{f}^{\mathrm{o}} 233, \mathrm{v}^{\mathrm{o}}\right)$

Au chapitre $\mathrm{V} \ll \mathrm{Du}$ propos du Prince en son plus court loisir $\gg$, l'évêque d'Auxerre recommande le trait d'esprit qui peut être doucement satirique, sans aller toutefois jusqu'à l'offense :

[... ] ceux qui ont anciennement emporté le prix de bien dire ont été aussi beaucoup estimés $[\ldots]$ pour sçavoir user à propos de quelque mot aigu et de gentille rencontre. En quoi tout Prince qui voudra suivre votre exemple se sçaura bien garder de poindre trop âprement. Car encore qu'un Roi puisse, non seulement dire, mais aussi faire tout ce qui lui plaît : si est-ce qu'en ceci où il cherche du plaisir il y doit avoir aussi quelque contentement pour ceux à qui il parle; de sorte que ses propos semblent plutôt chatouiller que piquer aigrement[.] (PER, p. 58)

C'est une fois de plus une leçon qu'Amyot a tirée de Plutarque qui considère lui aussi le « mot de rencontre » et le « traict » comme particulièrement appropriés à un gouvernant :

Il est bien vray que l'homme de gouvernement troussera bien aucunefois quelque mot de rencontre, et quelque traict de risee, mesmement si c'est pour chastier et 
provoquer quelqu'un modestement, et avec utilité, non pas le taxer ne picquer outrageusement en son honneur avec gaudisserie. (OM, $\left.\mathrm{f}^{\circ} 235, \mathrm{r}^{\circ}\right)$

Enfin, au chapitre IX « De quoi parle un Prince en temps d'affaires », le grand aumônier de France, après avoir fait valoir la supériorité du Prince capable de répondre lui-même à des ambassadeurs, insiste sur la nécessaire brièveté de l'éloquence royale, annonciatrice de la brevitas imperatoria que Guez de Balzac prêtera à Richelieu au siècle suivant ${ }^{34}$ :

Par quoi le Prince qui leur sait bien répondre en rapporte grande louange, non pas que je désire qu'il s'étende beaucoup en ceci ; car il y a moins de gravité en une longue oraison qu'en un parler bien serré[.] (PER, p. 75)

Plutarque formule la même exigence de brièveté, de condensation, chez celui qui manie les affaires d'État, en offrant Phocion comme modèle à imiter :

[ ... ] Demosthenes estoit bien un tresgrand orateur, mais $[\ldots]$ Phocion sçavoit mieux dire, pource que son langage en peu de paroles contenoit beaucoup de substance : et Demosthenes qui ne faisoit compte de tous les autres orateurs de son temps, quand Phocion se levoit pour parler apres luy : voilà, disoit-il, le coupperet de mes paroles qui se leve. (OM, fo $235 \mathrm{r}^{\mathrm{o}}$ et $\left.\mathrm{v}^{\mathrm{o}}\right)$

On pourrait multiplier ainsi les exemples qui démontreraient la place centrale occupée par le traité de Plutarque dans la réflexion d'Amyot sur l'éloquence royale. Il est cependant d'autres sources hellénistiques auxquelles le Projet de l'eloquence royale est redevable par-delà Plutarque et Denys d'Halicarnasse. Ainsi, à propos du chapitre XIII « De l'élite des mots, et de la liaison », Fumaroli avait déjà fait remarquer la dette d'Amyot envers le Peri hermeneias de Démétrios :

Transposant au service de la prose française l'essentiel du De Elocutione de Démétrius de Phalère $[. .$.$] Amyot ouvre la voie à un atticisme français tenant du style moyen$ (style orné de la délectation), mais par la seule qualité du choix des mots et de l'organisation euphonique de la phrase, et du style simple, mais relevé d'heureuses $\ll$ rencontres $3^{35}$.

Autant l'Avant-discours de Du Perron s'attachait à poser les conditions de possibilité d'une rhétorique royale à partir de l'héritage latin, autant le Projet de l'eloquence royale d'Amyot se donne pour ambition d'énoncer les préceptes généraux de cette parole éloquente du monarque, en faisant son miel de la tradition hellénistique en général et de Plutarque en particulier. 


\section{La Rhetorique françoise faicte particulierement pour le roy Henry 3 \\ de Germain Forget ou « un livre à part de ces figures et ornemens d'oraison $\gg d$ 'inspiration ramiste}

Le troisième et dernier discours académique, la Rhetorique françoise faicte particulierement pour le roy Henry 3, vient compléter les deux autres en offrant ce que Du Perron appelle des « exemples particuliers » (ADR, p. 768-769) susceptibles d'être imités et mis en pratique par le roi. D'une certaine manière, un tel ouvrage était appelé de ses vœux par Amyot dans son chapitre sur l'élocution où il écrivait : «Surtout les mots qui sont figurés embellissent et enrichissent le langage : et me semble, Sire, que devez commander qu'on vous fasse un livre à part de ces figures et ornements d'oraison, avec les exemples. » Or, ce livre à part des figures et ornements n'est connu que d'après deux manuscrits incomplets, l'un conservé à la Biblioteca estense de Modène et édité par Giulio Camus en $1887^{36}$ et l'autre inédit et conservé à la bibliothèque de Carpentras ${ }^{37}$. Seul ce dernier manuscrit mentionne le nom de l'auteur : Germain Forget, avocat au siège présidial d'Évreux, mort en 1618 et auteur d'ouvrages juridiques, d'une pastorale et de quelques œuvres de circonstance, dont le Panegiric, ou chant d'allegresse sur la venue du tres chrestien Henry troisiesme $(1574)^{38}$. Enfin, ce traité est le seul à offrir des éléments de critique interne qui permettent de le dater de 1579, puisque la majorité de ses citations sont tirées de tragédies de Robert Garnier toutes publiées en 1579 ou auparavant ${ }^{39}$.

Ce traité est influencé par la réforme ramiste de la rhétorique qui a consisté, comme on le sait, à repenser les liens entre rhétorique et dialectique, en retirant à la rhétorique l'invention et la disposition pour les confier à la dialectique, si bien que, pour Ramus, seules l'élocution et l'action faisaient partie de la rhétorique proprement dite. Cet héritage n'apparaît pas évident à première vue, ne serait-ce parce que l'auteur divise d'entrée de jeu la rhétorique en quatre parties : « Rhetorique est un art de bien et disertement parler pour persuader quelque chose. Elle a quatre parties en soy, l'invention, l'elocution, la pronunciation et l'action $\gg$ (RFA, p. 15). En $y$ regardant de plus près, toutefois, force est de constater que la prononciation et l'action ne forment en réalité qu'une seule et même partie, l'action, si bien que l'on se retrouve avec une rhétorique en trois parties : l'invention, l'élocution et l'action. Cela fait quand même une partie de plus que dans la Rhetorica (1548) d'Omer Talon ou, dans son pendant vernaculaire, la Rhetorique françoise (1555) d'Antoine Fouquelin, où ne sont traitées que l'élocution et l'action, qui forment l'envers et l'endroit d'une même médaille, vu que, dans la tradition cicéronienne, l'action est souvent assimilée à une sorte d'élocution du corps ${ }^{40}$. 
En lisant le chapitre consacré à l'invention, on s'aperçoit rapidement que cette partie est réduite comme peau de chagrin et, pour l'essentiel, renvoyée à la dialectique : « aussy semblent ils [=les lieux oratoires] apartenir plus particulierement a un dialecticien que non pas a un orateur $\gg(R F A, \mathrm{p} 19)$. De fait, Ramus considérait que l'invention et la disposition (qu'il appellait jugement) relevaient de la dialectique, titre de son célèbre traité de 1555. La prépondérance de l'élocution est évidente, dès lors que l'on tient compte de l'importance relative de chaque partie ${ }^{41}$ :

$$
\begin{array}{ll}
\text { Invention : } & 875 \text { mots } \\
\text { Élocution : } & 3331 \text { mots } \\
\text { Action : } & 1246 \text { mots }
\end{array}
$$

L'essentiel du traité est en fait constitué par le classement, la description et l'illustration des tropes et figures. Or, dans cette section, l'influence, sinon la dépendance à l'égard de la Rhetorica de Talon ou de la Rhetorique françoise de Fouquelin est évidente, si bien que l'on peut comprendre le titre du traité, Rhetorique françoise faicte particulierement pour le roy Henry 3 , comme la simple adaptation au destinataire

\begin{tabular}{|c|c|c|}
\hline $\begin{array}{c}\text { Germain Forget, } \text { Rhetorique } \\
\text { françoise faicte particuliere- } \\
\text { ment pour le roy Henry } 3 \text { (1579) }\end{array}$ & $\begin{array}{c}\text { Omer Talon, } \\
\text { Rhetorica (1548) }\end{array}$ & $\begin{array}{c}\text { Antoine Fouquelin, } \\
\text { Rhetorique françoise (1555) }\end{array}$ \\
\hline $\begin{array}{l}\text { Elocution est un lustre et orne- } \\
\text { ment d'oraison qui consiste en } \\
\text { tropes et figures. } \\
\text { Trope ou convertion est une } \\
\text { partie de l'elocution par lesquels } \\
\text { une diction est changée et muëe } \\
\text { de sa propre signification en une } \\
\text { autre. } \\
\text { Il y a quatre especes de tropes a } \\
\text { scavoir : Methaphore ou transla- } \\
\text { tion, Methonimie ou denomina- } \\
\text { tion, Sinecdoche ou comprehen- } \\
\text { tion et Ironie. (RFA, p. 19) }\end{array}$ & 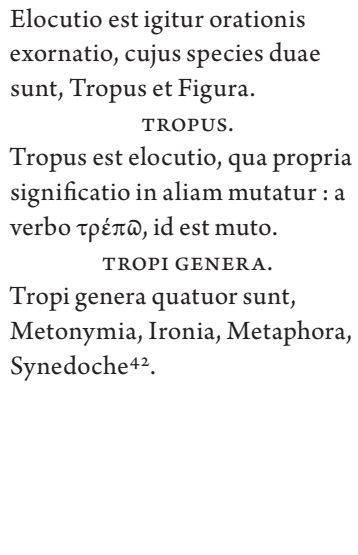 & $\begin{array}{l}\text { Elocution n'est autre chose, que } \\
\text { l'ornement et enrichissement de } \\
\text { la parole et oraison : laquelle a } \\
\text { deux espéces, l'une est appellée } \\
\text { Trope, l'autre Figure. } \\
\text { Trope. } \\
\text { Trope, est une elocution, par } \\
\text { laquelle la propre et naturelle } \\
\text { signification du mot es changée } \\
\text { en une autre : ce que declare } \\
\text { ce mot (Trope) qui signifie en } \\
\text { françois, mutation. } \\
\quad \text { Les especes de Trope. } \\
\text { Ily a quatre sortes de Trope : } \\
\text { Metonimie, Ironie, Metaphore, } \\
\text { Synecdoche } 43 \text {. }\end{array}$ \\
\hline
\end{tabular}
royal de la rhétorique ramiste. Pour s'en convaincre, il suffit de confronter l'ouverture de la section que chacun des trois traités consacre à l'élocution :

On pourrait ainsi multiplier les rapprochements qui montrent, à chaque fois, que le classement et la définition des procédés suivent le modèle ramiste. Cette influence ramiste, pour indéniable qu'elle soit, appelle cependant certaines nuances. 
Pour Alex Gordon, le traité est clairement ramiste, mais compile d'autres sources, notamment des rhétoriques latines scolaires ${ }^{44}$. Pour Kees Meerhoff, la Rhetorique françoise faicte particulierement pour le roy Henry 3 est certes ramiste dans son inspiration générale, mais s'éloigne d'une des notions centrales du ramisme, à savoir la fusion entre figure et nombre qui sont dissociés chez Forget. En outre, pour Meerhoff, il est peu probable que Forget ait utilisé la Rhetorique de Fouquelin. Il suppose plutôt le recours à la Rhetorica de Talon, parce que, d'une part, Forget ne reprend pas les exemples français de Fouquelin et que, d'autre part, dans le cas de la seconde espèce de métonymie, Forget donne un exemple latin, le célèbre vers d'Horace, « Pallida mors ... », donné aussi par Talon ${ }^{45}$.

Il reste à voir les exemples particuliers que donne cette rhétorique et qui la distinguent de la rhétorique de Fouquelin où les illustrations étaient tirées pour l'essentiel de Ronsard et des poètes de la Pléiade. Chez Forget, c'est le théâtre tragique de Robert Garnier (1544-1590) qui fournit l'essentiel des illustrations : Porcie (1568), Cornelie (1574), Marc Antoine (1578), Hippolyte (1573), La Troade (1579), Antigone (1579). Ces illustrations n'étaient sans doute pas destinées à nourrir l'imitatio du roi, ne serait-ce que parce que les vers n'ont pas leur place dans un discours politique. Mais les tragédies de Garnier ont sans doute été choisies, parce qu'elles font singulièrement écho à l'actualité politique et militaire du règne de Henri III, par exemple cette illustration de paronomase tirée de l'acte III de Cornelie :

$$
\begin{aligned}
& \text { Tel â par [s]on pouvoir moqueur } \\
& \text { Toute sa vie esté vainqueur, } \\
& \text { Qui au fort de sa gloire } \\
& \text { Pert contre unj[e]une belliqueur } \\
& \text { La vie et la victoire. (RFA, p. } 27 \text { ) }
\end{aligned}
$$

Les vers de Garnier ne sont toutefois pas les seules illustrations du traité. On trouve bon nombre de passages en prose qui, eux, étaient sans doute destinés à stimuler la pratique oratoire du roi parl'imitation, dans la mesure où ils n'auraient pas déparé une harangue politique prononcée, par exemple, devant les États Généraux. Nous en donnerons un seul exemple, assez long, mais qui mérite, pour sa beauté et sa véhémence, d'être cité in extenso. Il s'agit de l'illustration de la prosopopée :

Si vous autres princes qui furent si fam[e]us jadis, si devots, revenoint maintenant ; si ce bon S. Louys retournoit en sa France, qu'il seroit estonné de la voir heretique, de voir nos temples razés, nos monasteres embrazés, nos saints autels polus et contaminés, nos saints ornements profanés, que penseroit il, que diroit il, quel estonnement auroit il. Il m'est advis que j'entens ses propres [paroles] : Helas ! ma Frence, que tu es en miserable estat, que tu as bien changé de condition ; je t'ay vuë 
reluire en pieté et religion sur toutes les nations du monde, tu estois l'exemplaire de sainte[té] a tous peuples voisins, et maintenant tu as chassé Je[s]ucript ; et j'ay tant de fois gerroyé les payens, ay fondé tent de maisons de devotion, basti tant de sompt $[\mathrm{u}]$ eus temples, achepté si cherement les outils sacrés de notre redemption pour les abattre, detruire et profaner. (RFA, p. 33)

Après le résumé de la tradition rhétorique latine avec Du Perron, puis un sommaire de la réflexion hellénistique sur l'éloquence des gouvernants avec Amyot, il était logique que la boucle soit bouclée par une rhétorique qui accorde la primauté à l'elocutio dans le sillage de la révolution ramiste et qui prenne acte, du même coup, d'une des innovations majeures de la Renaissance en matière d'ars bene dicendi. C'est très précisément que ce fait Germain Forget, en s'appuyant sur la pensée de Ramus qui peut apparaître comme un des aboutissements de la réflexion humaniste en matière d'éloquence, dont les antécédents remontent jusqu'à Philippe Melanchthon et Rodolphe Agricola.

DANS LA DÉDICACE de sa traduction latine de la harangue de Henri III aux États Généraux de 1576, Benoît de Flandrois célèbre les qualités oratoires du dernier des Valois, en estimant qu'il se compare avantageusement à Isocrate par sa grâce (suavitas), à Lysias par sa justesse (subtilitas), à Hypéride par sa pénétration (acumen), à Démosthène par sa force (vis) et à Eschine par son éclat et la beauté de sa voix (sonitus et $\lambda \alpha \mu \pi \rho \circ \phi \varpi v i a){ }^{46}$, les cinq orateurs qui, de l'avis de Quintilien (X, 1, 76-80), résument les qualités maîtresses du style, ce qui était assez dire que Henri III était pourvu d'une nature éloquente, que, du reste, la plupart de ses contemporains reconnaissaient volontiers. Même Pierre de l'Estoile, si critique à l'égard du dernier des Valois, ne peut s'empêcher de relever, avec une certaine admiration, les mots « aigus et de gentille rencontre » de Henri III, par exemple lorsqu'en 1583 Guillaume Rose, évêque de Senlis et prédicateur du roi, ose prêcher en chaire contre le monarque, parce que ce dernier a couru les rues de Paris en masque le jour de Carême-Prenant :

Dequoy ledit Rose demanda pardon à Sa Majesté, laquelle, usant de sa bonté et douceur accoustumées, non seulement lui pardonna, mais, quelques jours apres, l'ayant envoyé querir, lui donna une assignation de 400 escus, pour acheter (lui dist le Roy) du sucre et du miel, pour aider à passer vostre quaresme et pour adoucir vos trop aspres et aigres paroles ${ }^{47}$.

Il restait à cultiver cette nature éloquente par le recours à l'ars rhetorica, un art rhétorique qui prenne appui sur tout le legs de l'Antiquité gréco-latine et de 
la Renaissance, de Cicéron, à Tacite, en passant par Quintilien, Plutarque, Denys d'Halicarnasse, Démétrios de Phalère et Pierre Ramus. C'est à cette tâche que les trois Académiciens du Palais se sont attelés avec succès, Du Perron exposant les possibilités de la rhétorique en contexte monarchique, Amyot résumant les préceptes généraux de l'éloquence royale et Germain Forget fournissant des exemples particuliers pour l'imitatio et l'exercitatio du roi, dans l'espoir qu'il tende vers l'idéal hiérarchique récapitulatif de tout cet héritage oratoire que Benoît de Flandrois résumera en une seule figure : 1'Hercule gaulois ${ }^{48}$.

\section{Notes}

1. Cet article est tiré d'une communication donnée au XVI ${ }^{\mathrm{e}}$ congrès de l'International Society for the History of Rhetoric qui s'est tenu à l'Université Marc-Bloch de Strasbourg en juillet 2007. Je remercie Marc André Bernier, Gary Ferguson, Pascale Fleury et Pierre Zoberman de leurs remarques et suggestions pour la rédaction de cette version revue et sensiblement augmentée. Pour les traités de l'Antiquité, nous avons pris le parti de citer les traductions françaises de l'Ancien Régime qui nous apparaissent beaucoup plus proches de l'épistémè des humanistes que les traductions modernes publiées dans la collection des Universités de France. En revanche, pour le texte latin, nous citons les éditions publiées aux Belles Lettres.

2. Jacques Amyot, Projet d'éloquence royale, préface de Philippe-Joseph Salazar, Paris, Les Belles Lettres, coll. « Le corps éloquent », 1992. Toutes les références ultérieures renverront à cette édition et seront précisées dans le corps du texte, entre parenthèses, précédées du sigle PER.

3. Jacques Davy Du Perron, « Traité de l'eloquence », dans L'art de parler. Anthologie des manuels d'éloquence, édition de Philippe-Joseph Salazar, Paris, Klincksieck, coll. « Cadratin », 2003, p. 134-145.

4. Robert J. Sealy, The Palace Academy of Henry III, Genève, Droz, coll. « Travaux d'Humanisme et Renaissance $\gg, 1981$, p. 153-166.

5. Sealy relève bien cette particularité, sans toutefois arriver à l'expliquer : «It is to be noted that with the exception of the last and unique set, on eloquence, the Academy during its entire life, from January 1576 to June 1579, was devoted exclusively to philosophy as that discipline was understood among the schoolmen as distinct from the liberal arts. » Robert J. Sealy, ouvr. cité, p. 163.

6. Voir, à ce propos, Pierre Chevallier, Henri III roi shakespearien, Paris, Fayard, 1985, p. $181-254$.

7. Jacques Amyot, Projet d'éloquence royale, ouvr. cité, p. 38, note 7 .

8. En l'absence de tout document d'archives, à l'exception des traités eux-mêmes, il est difficile de savoir si les auteurs avaient le dessein délibéré de couvrir chacun l'une des 
trois grandes traditions ici analysées. A posteriori, c'est la nette impression qui s'en dégage.

9. Si l'ordre de l'Académie du Palais avait suivi la chronologie courante qui veut que l'Antiquité grecque soit antérieure à l'Antiquité romaine, on se serait attendu à ce que la tradition grecque soit abordée (Amyot) avant la tradition latine (Du Perron), ce qui, d'après notre hypothèse, n’a pas été le cas, puisque le discours de Jacques Davy Du Perron, tout en résumant la tradition latine, est une introduction générale aux deux autres traités comme le prouve son titre d'avant-discours. Par ailleurs, le traité d'Amyot résume certes la tradition grecque, mais essentiellement celle de la période hellénistique, c'est-à-dire postérieure à Cicéron et Quintilien, si bien que, compte tenu de cette nuance, l'ordre ici reconstitué est bel et bien chronologique du point de vue du contenu même des traités.

10. Georges Grente et Michel Simonin, « Du Perron (cardinal Jacques Davy) », dans Michel Simonin (sous la dir. de), Dictionnaire des lettres françaises. Le XVI ${ }^{e}$ siècle, Paris, Fayard, coll. « La Pochothèque », 2001, p. 434-436.

11. Pierre de l'Estoile, Registre-Journal du règne de Henri III, édité avec une introduction et des notes par Madeleine Lazard et Gilbert Schrenck, Genève, Droz, coll. « Textes littéraires français $\gg, 2000$, tome IV, p. 105 .

12. Jacques Chevallier, ouvr. cité, p. 391.

13. Robert J. Sealy, ouvr. cité, p. 154. C'est René Radouant qui aurait repéré cette édition. Voir Guillaume Du Vair, De l'eloquence françoise, édition de René Radouant, Paris et Genève, Droz, 1970, p. 4.

14. Tacite, Dialogue sur les orateurs, [ ... ] par M. de S[igrais] de l'Académie Royale des Inscriptions et Belles-Lettres, Paris, De l'imprimerie de Monsieur, 1782, p. 1. Voir aussi Tacite, Dialogue des orateurs, texte établi par Henri Goezler et traduit par Henri Bornecque, Paris, Les Belles Lettres, collection des Universités de France, 1985, p. 24.

15. Tacite, Dialogue sur les orateurs, [ ... ] par M. de S[igrais], ouvr. cité, p. 86-87. Voir aussi Tacite, Dialogue des orateurs, texte établi par Henri Goezler et traduit par Henri Bornecque, ouvr. cité, p. 65.

16. Jacques Davy Du Perron, «Avant-Discours de Rhetorique, ou Traitté de l'eloquence $\gg$, dans Euvres diverses, Paris, Pierre Chaudiere, 1633, p. 759. Toutes les références ultérieures renverront à cette édition et seront précisées dans le corps du texte, entre parenthèses, précédées du sigle $A D R$.

17. Marc Fumaroli, L'Âge de l'éloquence. Rhétorique et « res literaria » de la Renaissance au seuil de l'époque classique [1980], Paris, Albin Michel, coll. «Bibliothèque de l'Évolution de l'Humanité $\gg, n^{\circ} 4$, 1994, p. 494. Fumaroli renvoie ici au livre I, chapitre 41, des Essais de Montaigne et au livre II des Recherches de la France de Pasquier.

18. Dans l' «Élégie à G. Des-Autels », Ronsard formule la poétique des Discours des Miseres de ce temps, en affirmant vouloir conserver les provinces du royaume de France dans le giron du catholicisme « par livres » et « defendre nos maisons, / Non par le fer trenchant ains par vives raisons $\gg$. Ce faisant, il cherche à mettre en avant une alternative à la violence, mais aussi à reconquérir le terrain perdu par les catholiques au 
profit des huguenots sur le plan de l'éloquence. Ronsard, Euvres complètes, édition de Jean Céard, Daniel Ménager et Michel Simonin, Paris, Gallimard, coll. « Bibliothèque de la Pléiade », 1994, tome II, p. 1011, v. 12 et 15-16.

19. Quintilien, Institution oratoire, édition de Jean Cousin, Paris, Les Belles Lettres, collection des Universités de France, 1976, tome II, p. 88. Voici la traduction française de 1718 de l'abbé Gédoyn : « [ ... ] de travailler leurs nids avec tant d'adresse et d'industrie, de prendre tous les soins qu' ils prennent de leurs petits, de faire des ouvrages inimitables, comme sont la cire et le miel, de s'assurer mesme des provisions pour la nécessité ; c'est peut-estre l'effet d'une sorte de raison. Mais parce qu'ils ne parlent point, tout ce qu'ils font de plus surprenant ne les tire point du rang des bestes. Enfin représentons-nous un muet; que fait en luy cet esprit celeste qui l'anime? Et que le secours qu'il en reçoit est foible! » Quintilien, De l'Institution de l'orateur traduit par M. l'abbé Gédoyn, Chanoine de Sainte Chapelle de Paris, de l'Académie Royale des Inscriptions, et belles Lettres, Paris, Grégoire Dupuis, 1718, p. 130-131.

20. Cicéron, De l'orateur, édition d'Edmond Courbaud, Paris, Les Belles Lettres, collection des Universités de France, 1957, tome I, p. 10. Voici la traduction française de $1642: \ll[\ldots]$ combien d'excellents Politiques avons-nous veu paroitre dans le Senat? Il y en avoit davantage du tems de nos Peres, au lieu que nous parcourons plusieurs siecles sans y trouver un bon Orateur, et qu'à peine dans chaque âge en pouvonsnous découvrir un seul qui ait esté mediocre $\gg$ La Rhétorique de Cicéron ou les trois livres du dialogue de l'orateur en latin et en françois, Lyon, Horace Moulin, 1642, p. 6.

21. Cette traduction correspond au texte latin suivant : « in forensibus disceptationibus judiciorum, aut deliberationum » (Cicéron, De l'orateur, édition d'Edmond Courbaud, ouvr. cité, p. 15). Pour la traduction, nous citons La Rhétorique de Cicéron ou les trois livres du dialogue de l'orateur en latin et en françois, ouvr. cité, p. 15.

22. Lettres de Henri III, roi de France, recueillies par Pierre Champion et publiées par Michel François et Jacqueline Boucher, Paris, Klincksieck et Honoré Champion, 19592006, 6 tomes publiés à ce jour.

23. Quintilien, Institution oratoire, édition de Jean Cousin, ouvr. cité, tome II, p. 93. Voici la traduction de l'abbé Gédoyn : « Quand Annibal, par exemple, se voyant enfermé par Fabius, fit attacher des fascines de sarment aux cornes de quantité de beufs, commanda qu'on y mist le feu, et qu'on chassast ces animaux vers les hauteurs, pour faire croire à Fabius qu'il décampoit, il sçavoit fort bien ce qu'il faisoit. » Quintilien, De l'Institution de l'orateur traduit par M. l'abbé Gédoyn, ouvr. cité, p. 134.

24. Quintilien, Institution oratoire, édition de Jean Cousin, ouvr. cité, tome II, p. 95. Voici la traduction de l'abbé Gédoyn : «Un pilote veut arriver heureusement au port, son vaisseau devient le joüet de la tempeste et des vents ; en est-il moins bon pilote? Il n'abandonne point le gouvernail, le reste est entre les mains des Dieux. Un Médecin s'applique à la guérison d'un malade, cependant une complexion foible, la violence du mal, un accident imprévû rendent ses soins inutiles. L’a-t-il traité dans les regles? il a satisfait à son art. » Quintilien, De l'Institution de l'orateur traduit par M. l'abbé Gédoyn, ouvr. cité, p. 135 . 
25. Quintilien, Institution oratoire, édition de Jean Cousin, ouvr. cité, tome II, p. 102. Voici la traduction de l'abbé Gédoyn : «Si Praxitele s'estoit efforcé de faire une statuë d'une de ces pierres, dont on fait les meules de moulin, j'aimerois mieux sans comparaison un beau marbre tout brute ; mais s'il avoit mis ce beau marbre en œuvre, il auroit fait quelque chose de fort précieux, et qui tireroit son prix des mains de l'ouvrier, bien plus que de sa propre beauté. » Quintilien, De l'Institution de l'orateur traduit par M. l'abbé Gédoyn, ouvr. cité, p. 140.

26. Quintilien, Institution oratoire, édition de Jean Cousin, ouvr. cité, tome II, p. 100. Voici la traduction de l'abbé Gédoyn : «Il y a plusieurs sortes d'Arts, les uns purement spéculatifs se renferment dans la théorie, c'est-à-dire dans la connoissance et la considération de leur objet, telle est l'Astrologie, qui sans autre action se borne à l'intelligence des choses celestes, dont elle fait sa principale estude : les autres à qui l'on donne le nom de pratiques, consistent dans l'action. Elles en font leur fin, leur mérite et leur perfection ; mais c'est une action qui ne laisse rien après elle, comme la danse. Les autres enfin qu'on peut appeller effectifs, se terminant à un effet qui est un ouvrage sensible et permanent, telle est la peinture. // Il est à croire que la Rhétorique est du nombre des seconds ; car c'est par l'action qu'elle remplit ses devoirs, et tous les maistres en jugent ainsi. Il me semble néanmoins qu'elle tient aussi beaucoup des deux autres[.] » Quintilien, De l'Institution de l'orateur traduit par M. l'abbé Gédoyn, ouvr. cité, p. 139.

27. Quintilien, Institution oratoire, édition de Jean Cousin, ouvr. cité, tome II, p. 77. Voici la traduction de l'abbé Gédoyn : « Lors qu'Antoine plaidant pour M. Aquilius, par un mouvement imprévû déchira l'habit de l'accusé, et fit voir les blessures qu'il avoit reçûës en combattant pour la patrie, se fia-t-il à la force de ses raisons? Non, mais il arracha des larmes au peuple Romain, qui ne put résister à un spectacle si touchant, et renvoya le criminel absous. [ ... ] Enfin nous avons l'exemple de Phryné, que sa beauté deffendit mieux que toute l'éloquence d'Hyperide ; car tout grand Orateur qu'il estoit, il alloit perdre sa cause, lorsqu'entr'ouvrant tout à coup la robbe de cette belle accusée, il fit sentir aux juges de l'A réopage, qu'elle pouvoit les charmer comme les autres. » Quintilien, De l'Institution de l'orateur traduit par M. l'abbé Gédoyn, ouvr. cité, p. 122-123.

28. Projet de l'eloquence royale, composé pour Henry III, roi de France, par Jacques Amyot, Évêque d'Auxerre, Grand Aumônier de France, etc. D'après le Manuscrit autographe de l'Auteur, Versailles et Paris, Imprimerie de Ph.-D. Pierres et Chez Lamy, Libraire, 1805 .

29. Jacques Chevallier, ouvr. cité, p. 391.

30. Alexandre Cioranescu et Robert Aulotte, « Aymot (Jacques) », dans Michel Simonin (sous la dir. de), Dictionnaire des lettres françaises. Le XVI ${ }^{e}$ siècle, Paris, Fayard, coll. «La Pochothèque », 2001, p. 55-57.

31. Quintilien, De l'Institution de l'orateur traduit par M. l'abbé Gédoyn, ouvr. cité, p. 66. Cette traduction correspond au texte latin suivant : « orbis ille doctrinae, quem 
Graeci encyclion paedian vocant ». Quintilien, Institution oratoire, édition de Jean Cousin, ouvr. cité, 1975, tome I, p. 130.

32. Euvres morales et meslees de Plutarque, Translatees de Grec en François, reveuës et corrigees en plusieurs passages par Messire Jacques Amyot Evesque d'Auxerre, Grand Aumonier de France [1572], Anvers, Nicolas Soolmans, 1576, fo 234, $\mathrm{r}^{\mathrm{o}}$. Toutes les références ultérieures renverront à cette édition et seront précisées dans le corps du texte, entre parenthèses, précédées du sigle $O M$.

33. Denys d'Halicarnasse, Opuscules rhétoriques, texte établi et traduit par Germaine Aujac, Paris, Les Belles Lettres, collection des Universités de France, 1978, tome I, p. 71.

34. Voir à ce propos Philippe-Joseph Salazar, «Préface. Le monarque orateur », dans Jacques Amyot, Projet d'éloquence royale, ouvr. cité, p. 23.

35. Marc Fumaroli, ouvr. cité, p. 496.

36. Precetti di rettorica scritti per Enrico III re di Francia, édition de Giulio Camus, Modène, Antica Tipografia Soliani, 1887. Toutes les références ultérieures renverront à cette édition et seront précisées dans le corps du texte, entre parenthèses, précédées du sigle RFA.

37. Voir, à ce propos, Raymond Lebègue, « La tragédie française au XVI ${ }^{\mathrm{e}}$ siècle $\gg, R e$ vue des cours et conférences, XXXIII, $\mathrm{n}^{\circ}$ 2, 30 juin 1932, p. 541-542; Alex L. Gordon, Ronsard et la rhétorique, Genève, Droz, 1970, p. 16-18; et Alex L. Gordon, « Ramist Influence in Germain Forget's Rhétorique françoise faicte particulièrement pour le Roy Henry Troiziesme (1583) », Romance Notes, XXIII, 1982-1983, p. 238-263.

38. Michel Simonin, « Forget, Germain », dans Michel Simonin (sous la dir. de), Dictionnaire des lettres françaises. Le XVI ${ }^{e}$ siècle, Paris, Fayard, coll. « La Pochothèque », 2001, p. 523-524.

39. Voir, à ce propos, Robert J. Sealy, ouvr. cité, p. 158-159 et l'introduction de Giulio Camus, dans Precetti, ouvr. cité, p. 12-13.

40. Dans le De Oratore (III, 222), Cicéron écrit que « l'Action [ ... ] est appellée l'Eloquence du corps $\gg$ (Est enim quasi sermo corporis) et, dans l'Orator (55), que « [1'] action est comme l'élocution du corps $\gg$ (Est enim actio corporis quaedam eloquentia). La Rhétorique de Cicéron ou les trois livres du dialogue de l'orateur en latin et en françois, ouvr. cité, p. 610 ; Cicéron, De l'orateur, édition de Henri Bornecque et Edmond Courbaud, ouvr. cité, tome III, 1956, p. 94 ; et Cicéron, L'Orateur, édition d'Albert Yon, Paris, Les Belles Lettres, collection des Universités de France, 1964, p. 20.

41. Deux autres parties, qui ne sont pas annoncées dans l'introduction, sont ajoutées à la fin du traité : « Des nombres d'oraison » (661 mots) et « De l'exorde » (946 mots).

42. Omer Talon, Rhetorica ad Carolum Lotharingum Cardinalem Guisianum [1548], Paris, Mathieu David, 1549, aiii, vo.

43. Antoine Fouquelin, Rhetorique françoise [1555], Paris, André Wechel, 1557, Aiiii, vo et $\mathrm{Av}, \mathrm{r}^{\mathrm{o}}$.

44. Alex L. Gordon, « Ramist Influence in Germain Forget's Rhétorique françoise faicte particulièrement pour le Roy Henry Troiziesme (1583) », art. cité, p. 262. 
45. Kees Meerhoff, Rhétorique et poétique au XVI siècle : Du Bellay, Ramus et les autres, Leyde, E. J. Brill, 1986, p. 257-261.

46. $\ll[\ldots]$ foelicissime omnes omnium oratorum laudes consecutus fuit, non suavitate Isocrati, non subtilitate Lysiae, non acumine Hyperidi, non vi Demostheni, non sonitu et $\lambda \alpha \mu \pi \rho \circ \phi \pi v i \alpha$ ipsi concedens Aeschini : Quibus etiam Salomonem, Orpheum, Amphionem, et Gallicum illum Herculem aequare una omnium voce atque sententia existimatus fuit. » Oratio Henrici III. Galliae ac Poloniae Regis, gallice, summa cum actionis dignitate Comitiis habita, in praeclara et Regum altrice Blesiorum urbe: Ad tres Gallici populi praecipuos ordines, 1576. octavo Idus Decembr., Paris, Federic Morel, 1577, Aii, $v^{0}$. Ce passage paraphrase le De oratore (III, 28) de Cicéron. Pour la traduction française des termes techniques, nous avons suivi La Rhétorique de Cicéron ou les trois livres du dialogue de l'orateur en latin et en françois, ouvr. cité, p. 478. Le terme de $\lambda \alpha \mu \pi \rho \circ \phi \pi v i ́ \alpha$ ne se trouve pas chez Cicéron, mais est attesté chez Hérodote (VI, 60). Nous nous sommes inspiré de la traduction d'Andrée Barguet dans Hérodote et Thucydide, Euvres complètes, introduction de Jacqueline de Romilly, Paris, Gallimard, coll. «Bibliothèque de la Pléiade », 1964, p. 428.

47. Pierre de l'Estoile, Registre-Journal du règne de Henri III, ouvr. cité, tome IV, p. 74.

48. Voir, à ce propos, notre article « Henri III, nouvel Hercule gaulois », dans Laurent Pernot (sous la dir. de), New Chapters in the History of Rhetoric, Leyde, E. J. Brill, à paraître. Le même collectif paraîtra en traduction bulgare aux Presses universitaires de Sofia. 